\title{
Diagnostic Phase of Calcium Scoring Scan Applied as the Center of Acquisition Window of Coronary Computed Tomography Angiography Improves Image Quality in Minimal Acquisition Window Scan (Target CTA Mode) Using the Second Generation 320-Row CT
}

\author{
Eriko Maeda, ${ }^{1}$ Kodai Yamamoto, ${ }^{1}$ Shigeaki Kanno, ${ }^{1}$ Kenji Ino, ${ }^{2}$ Nobuo Tomizawa, ${ }^{3}$ \\ Masaaki Akahane, ${ }^{4}$ Rumiko Torigoe, ${ }^{5}$ and Kuni Ohtomo ${ }^{1}$ \\ ${ }^{1}$ Department of Radiology, Graduate School of Medicine, University of Tokyo, 7-3-1 Hongo, Bunkyo-ku, Tokyo 113-8655, Japan \\ ${ }^{2}$ Imaging Center, The University of Tokyo Hospital, 7-3-1 Hongo, Bunkyo-ku, Tokyo 113-8655, Japan \\ ${ }^{3}$ Department of Radiology, New Tokyo Hospital, 1271 Wanagaya, Matsudo, Chiba 270-2232, Japan \\ ${ }^{4}$ Department of Radiology, NTT Medical Center Tokyo, 5-9-22 Higashi-Gotanda, Shinagawa-ku, Tokyo 141-8625, Japan \\ ${ }^{5}$ Toshiba Medical Systems Corporation, Tokyo Metropolitan Regional Office, 1-6 Tsukuda 2-Chome, Chuo-ku, Tokyo 104-0051, Japan
}

Correspondence should be addressed to Eriko Maeda; emaeda-tky@umin.ac.jp

Received 31 August 2015; Revised 4 January 2016; Accepted 13 January 2016

Academic Editor: Jinyuan Zhou

Copyright (C) 2016 Eriko Maeda et al. This is an open access article distributed under the Creative Commons Attribution License, which permits unrestricted use, distribution, and reproduction in any medium, provided the original work is properly cited.

\begin{abstract}
Objective. To compare the image quality of coronary computed tomography angiography (CCTA) acquired under two conditions: $75 \%$ fixed as the acquisition window center (Group 75\%) and the diagnostic phase for calcium scoring scan as the center (CS; Group CS). Methods. 320-row cardiac CT with a minimal acquisition window (scanned using "Target CTA" mode) was performed on 81 patients. In Group 75\% ( $n=40)$, CS was obtained and reconstructed at 75\% and the center of the CCTA acquisition window was set at $75 \%$. In Group CS ( $n=41)$, CS was obtained at $75 \%$ and the diagnostic phase showing minimal artifacts was applied as the center of the CCTA acquisition window. Image quality was evaluated using a four-point scale (4-excellent) and the mean scores were compared between groups. Results. The CCTA scan diagnostic phase occurred significantly earlier in CS $(75.7 \pm 3.2 \%$ vs. 73.6 $\pm 4.5 \%$ for Groups $75 \%$ and CS, resp., $p=0.013)$. The mean Group CS image quality score $(3.58 \pm 0.63)$ was also higher than that for Group $75 \%$ (3.19 $\pm 0.66, p<0.0001)$. Conclusions. The image quality of CCTA in Target CTA mode was significantly better when the center of acquisition window is adjusted using CS.
\end{abstract}

\section{Introduction}

Adult coronary computed tomography angiography (CCTA) usually begins with a noncontrast electrocardiogram-gated chest CT called a "calcium scoring (CS) scan" performed after scout scans. CS is used to determine the range of the CCTA scan and to calculate an Agatston score, the counterpart to the calcium score which is obtained using electron beam CT [1]. Because evaluation of the coronary lumen during CCTA is hampered by dense calcification of the coronary artery wall, the Agatston score can be used to select cases with diffuse coronary calcifications, who should not receive further scans due to the likelihood of limited benefit and the risks associated with contrast material and additional radiation exposure [2-6].

Second generation 320-row CT scanners with a rotation speed of $275 \mathrm{~ms}$ can scan the whole heart in one rotation, using a minimal acquisition window ("Target CTA"; Toshiba, Tochigi, Japan). This scan mode can be applied for evaluating cases with a heart rate lower than approximately 75 beats per minute (bpm). With Target CTA scans, the center of the acquisition window is set to any integral percentage, and 
X-ray exposure is limited to only the minimum duration needed to reconstruct the images. Although the acquisition window is set using only one integral, such as $75 \%$, the scan has a short reconstruction window and the diagnostic phase (i.e., the phase showing minimal artifacts) can be searched for within the acquisition window. As an example, use of a Target CTA scan of $75 \%$ in a patient with an RR interval of $1000 \mathrm{~ms}$ results in an acquisition window of $689-811 \mathrm{~ms}$, with the center of the acquisition window at $750 \mathrm{~ms}$ and the width of reconstruction window at $122 \mathrm{~ms}$ (note: the acquisition window or the exposure duration always exceeds the reconstruction window). These phase types are searched using "PhaseNavi" cardiac-phase search software (Toshiba, Tochigi, Japan), which automatically searches for the phase that produces the lowest average SD value for all voxels in the volume. However, the results of automated phase searching do not always correspond with the diagnostic phase. Also, the most static phase needs to be visually searched using the same software, if the coronary arteries contain motion artifacts at the point identified by the automated phase search. Compared with other methods, Target CTA is reported to produce low-dose scans together with noninferiority in image quality $[7,8]$. The value of $75 \%$ is widely used as the center of the Target CTA acquisition window, although this value is empirical [9-13].

CS has been scanned using a 75\% Target CTA mode for patients with a heart rate $(\mathrm{HR}) \leq 75 \mathrm{bpm}$, and at $40 \%$ for those with an HR $>75 \mathrm{bpm}$. During the CS Target CTA scan, the reconstruction phase was fixed to $75 \%$, and it was not possible for PhaseNavi software to adjust the reconstruction phase. However, a recent software upgrade (Aquilion ONE ViSION edition version 6.0; Toshiba, Tochigi, Japan) allows for the adjustment of CS scan reconstruction window. We hypothesized that the CS diagnostic phase correlates with that of CCTA and that the image quality of CCTA would improve with adjustment of the center of the CCTA acquisition window using the CS diagnostic phase as compared to using a fixed percentage value.

Therefore the aim of this study was to determine potential correlation between CS and CCTA scan diagnostic phases and to compare the CCTA image quality with the use of $75 \%$ (Group 75\%) versus the CS diagnostic phase (Group CS) as the center of the acquisition window.

\section{Materials and Methods}

This study which was conducted at a single research center was approved by the local ethics committee. Because of this study's retrospective design, the requirement for informed consent prior to study participation was waived.

2.1. Patients. The Target CTA scan was applied to the patients with sinus rhythm and an $\mathrm{HR} \leq 75 \mathrm{bpm}$. For patients with arrhythmias, a different acquisition program had to be applied in order to run an arrhythmia exclusion program. Thus patients with sinus rhythm and an $\mathrm{HR} \leq 75 \mathrm{bpm}$ were included in our study.
We retrospectively reviewed the records of 162 consecutive patients who underwent CCTA between October 2013 and February 2014. In December 2013, we started to adjust the center of the CCTA acquisition window for Target CTA using the CS diagnostic phase. Single volume Target CTA mode scanning was not used for 81 patients because of the following reasons: nonsinus rhythm $(n=13)$; single beat scan with long acquisition window because of heart rate fluctuation $(n=$ $17)$; multiple heart beat acquisition $(n=23)$; wide-volume scanning performed to evaluate bypass grafts or aorta $(n=$ 16 ); ventricular evaluation prior to catheter ablation $(n=$ 3); or irregular protocol for evaluation of complex cardiac anomaly $(n=9)$. The final study group included 81 patients (Group 75\%, $n=41$; Group CS, $n=40$ ) who were scanned because of known or suspected coronary artery disease with chest pain and/or dyspnea, or abnormal electrocardiogram, echocardiogram, or treadmill results.

2.2. CT Data Acquisition. All patients underwent CT angiography performed using second generation 320detector row CT for all enrolled patients and prospective electrocardiogram-gated scans were performed in one heartbeat. The scanning parameters were as follows: detector configuration, $320 \times 0.5 \mathrm{~mm}$; gantry rotation time $275 \mathrm{~ms}$; and tube potential, $120 \mathrm{kVp}$. The tube current was set at $150 \mathrm{~mA}$ for calcium scoring scan and from $250 \mathrm{~mA}$ to $760 \mathrm{~mA}$ for CCTA depending on patient body weight. The mean effective dose was derived from the dose length product multiplied by a conversion coefficient for the chest $\left(\kappa=0.014 \mathrm{mSv} \times \mathrm{mGy}^{-1} \times \mathrm{cm}^{-1}\right)$ [14]. The scan length ranged from 12 to $16 \mathrm{~cm}$ depending on the size of the heart.

For CS, the center of the acquisition window was set at 75\% throughout the period. Until December 2013, the CS reconstruction phase was not adjustable. After December 2013, the reconstruction phase became adjustable, allowing the diagnostic phase with minimal artifacts to be determined at the CT console using PhaseNavi software. The reconstructed slice thickness was $1.0 \mathrm{~mm}$ with a $1.0 \mathrm{~mm}$ increment. Images were reconstructed using a "medium soft tissue" kernel (FC04). We routinely use this low-pass kernel for cardiac CT because it reduces beam hardening artifacts originating from the vertebra and the aorta.

For CCTA, the center of the acquisition window was empirically fixed at $75 \%$ of the RR interval until November 2013 (Group 75\%). After December 2013, the center of acquisition window was set at the CS diagnostic phase value (Group CS). For CCTA scans in both groups, the phase with minimum artifacts was determined at the CT console using PhaseNavi software. Half cycle reconstruction was performed for all patients, meaning that there was a full cycle of X-ray exposure but only a half cycle of data was used for reconstruction. The reconstructed slice thickness was $0.50 \mathrm{~mm}$ with an increment of $0.25 \mathrm{~mm}$. Images were reconstructed using a "medium soft tissue" kernel (FC04) with Adaptive Iterative Dose Reduction in 3D (AIDR-3D) strong and symmetric cone beam reconstruction $[15,16]$. Images were transferred to a workstation (ZIO Station System; Ziosoft, Tokyo, Japan) for processing. 
Patients received $22.2 \mathrm{mg} \mathrm{I} / \mathrm{kg} / \mathrm{s}$ of iopamidol $370 \mathrm{mg} \mathrm{I} /$ $\mathrm{mL}$ (Iopamiron 370; Bayer, Osaka, Japan). Contrast medium was injected for $10 \mathrm{sec}$ and then a 50:50 mixed contrast medium and saline for $4 \mathrm{sec}$, followed by a $30 \mathrm{~mL}$ saline flush. Bolus tracking in the ascending aorta was performed using a double threshold of 100 and 260 Hounsfield Units (HU). Patients were assigned to breathe in and hold their breath after the first threshold. The scan started just after the second threshold.

Nineteen patients were being treated with an oral $\beta$ blocker (e.g., bisoprolol and carvedilol) as a part of their baseline medication. An oral $\beta$-blocker (20-40 mg of metoprolol) was administered to 18 patients with HR higher than $>65 \mathrm{bpm}$. The patients were told to take the medicine 2 hours prior to CT angiography. Landiolol (Corebeta; Ono Pharmaceutical, Osaka, Japan) was administered intravenously at $0.125 \mathrm{mg} / \mathrm{kg}$ when a patient's HR was over $75 \mathrm{bpm}$ during the time between the calcium scoring scan and CCTA. Patients underwent CCTA 4-7 min after injection $(n=11)$. No patient had any contraindication preventing $\beta$-blocker use, and no $\beta$-blocker side effects were observed or reported. All patients received $2.5 \mathrm{mg}$ sublingual isosorbide dinitrate (Nitorol; Eisai, Tokyo, Japan) before imaging.

2.3. Subjective Image Analysis. Subjective image quality was rated by Kodai Yamamoto and Eriko Maeda, two cardiovascular radiologists with 5 and 11 years of experience, respectively. Both were blinded from the details of the CT data sets, provided in a randomized order, and clinical information. The Society of Cardiovascular Computed Tomography 18segment classification was applied for the analysis of coronary angiography data [17]. Image quality was graded on a persegment level, and a study was deemed diagnostic when every anatomically present segment $(\geq 1.5 \mathrm{~mm})$ could be assessed for the presence of atherosclerosis and severity of stenosis. The results were scored according to a four-point scale as previously described: 4 , excellent, no artifact; 3 , good, mild artifact; 2, acceptable, moderate artifact present, but images still interpretable; 1 , unable to evaluate, severe artifact making interpretation impossible [18]. When scores differed between the two readers, the final score was determined by review and consensus.

2.4. Objective Image Analysis. Regions of interest (ROIs) were drawn on a cross-sectional image, at the proximal ascending aorta; the proximal, middle, and distal segments of the right coronary; the left anterior descending artery; and the left circumflex artery. The average CT number (in HU) and noise were recorded for each segment using a circular ROI. The ROI was made as large as possible while carefully avoiding inclusion of the vessel wall to prevent partial volume effects (Figure 1). An ROI was placed immediately next to the vessel contour on an axial image and the average CT number was recorded. The overall signal-to-noise ratio was defined as the average standard deviation of the circular ROI placed at the ascending aorta. The SNR of each coronary vessel was defined as the average standard deviation of the circular ROI placed at the proximal, middle, and distal segments of the vessel. The overall contrast-to-noise ratio (CNR) was calculated as the difference in the CT number between the ascending aortic lumen and nearby connective tissue divided by the overall image noise. For each coronary vessel, CNR was defined as the average CNR of the circular ROI placed at the proximal, middle, and distal segments of the vessel. We expected that the ascending aorta SNR and CNR would not change between the groups, because aortic image noise is unlikely to be related to the motion of the coronary arteries. Therefore we calculated SNR and CNR at the ascending aorta as a control.

2.5. Statistical Analysis. A power analysis was performed to determine the minimal cohort size required using $\mathrm{G}^{*}$ power version 3.1.9.2. (Universitat Düsseldorf, Düsseldorf, Germany). Our hypothesis was that per-segment subjective image quality would improve in Group CS. To detect a difference of 0.1 in subjective image quality score, the minimum sample size was determined to be a total of 527 segments (approximately 30 patients) at 0.90 power. Sample size calculations were based on a type- 2 error $(\alpha=)$ of 0.05 [19].

The minimal acquisition window scans (Target CTA mode) for October 2013 were reviewed $(n=20)$ and the reconstruction window was calculated from the console information. The reconstruction window was invariably proven to be $122 \mathrm{~ms}$. To know the percentage of patients in Group CS whose best reconstruction phase would not have been included in the scan if the fixed $75 \%$ scan was applied, we compared the actual exposure time as well as "virtual" $75 \%$ exposure \{i.e., $[R R$ interval $(\mathrm{ms}) \times 0.75] \pm 61 \mathrm{~ms}\}$. The correlation between the CS and CCTA scan diagnostic phases was calculated using Spearman's correlation coefficient analysis.

All statistical analyses were performed using JMP software (version 10; SAS, Cary, NC, USA). Quantitative variables were expressed as the mean \pm standard deviation and group differences were tested by Student's $t$-test. Categorical values were expressed as the number (percentage) and were compared using Fisher's exact test or the chi-squared test. Statistical significance was accepted when $p<0.05$.

\section{Results}

There was no group difference in patient demographics and scanning parameters (Tables 1 and 2). The mean best reconstruction phase (\%) for the CCTA scan was significantly earlier for Group CS, although quite widely variable range was observed among the diagnostic phases (Table 3, Figure 2). For eight patients in Group CS, the diagnostic phase occurred outside of the virtual 75\% exposure (19.5\%) (Figure 3). A significant correlation was detected between CS and CCTA diagnostic phases (Spearman's correlation coefficient 0.351, $p=0.02, R^{2}=0.113$ ).

Among the 1458 total segments captured for each group (18 segments in 81 patients), 137 segments were not evaluable because the segment was absent or too small (60 and 77 segments for Group 75\% and Group CS, resp.). The subjective image quality scores were significantly better in Group CS, both for overall and for branch specific analyses (Table 4). 


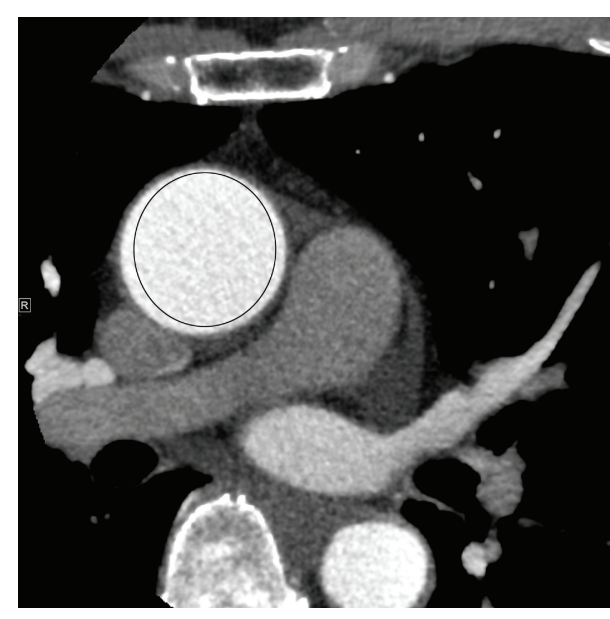

(a)

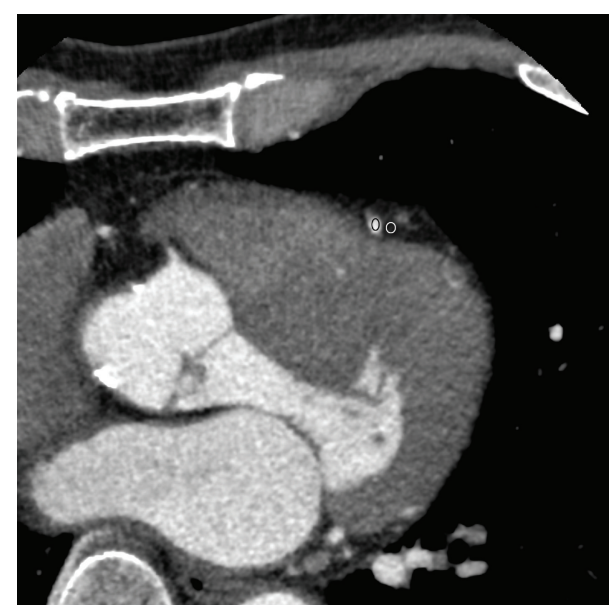

(c)

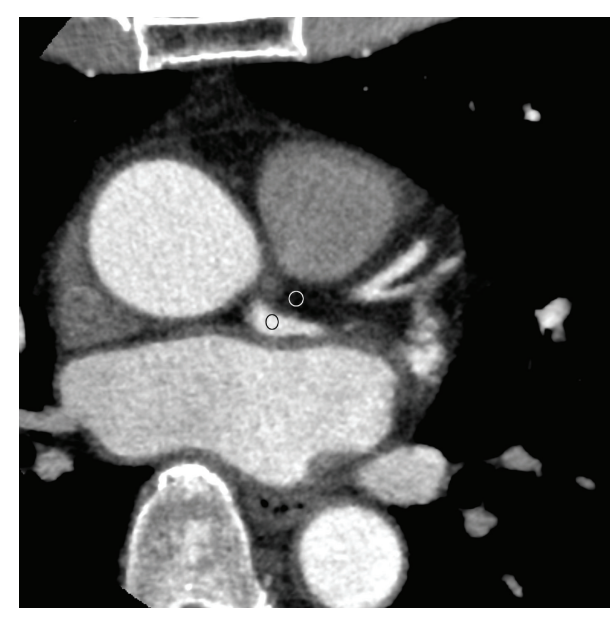

(b)

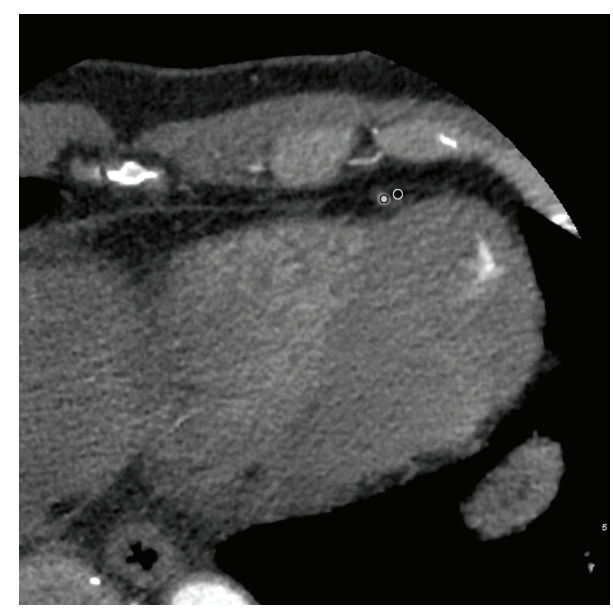

(d)

FIGURE 1: Examples of regions of interest (ROIs) drawn on a cross-sectional image, at the proximal ascending aorta; the proximal, middle, and distal segments of the left anterior descending artery. Black circle shows ROIs drawn inside the lumens of the arteries, while white circle shows ROIs drawn in the nearby connective tissue to calculate contrast-to-noise ratio.

Branch specific analyses of objective image quality scores were also higher in Group CS (Table 5). Interobserver agreement on subjective image quality was "good" $(\kappa=$ $0.68)$. When patients with poor interobserver agreement were defined as "difference in subjective score between two graders being 2 or more in more than five segments," we found that just four patients qualified as having poor interobserver agreement due to either the presence of dense calcification or multiple stents.

\section{Discussion}

This is the first report on the use of the CS diagnostic phase as the center of the CCTA acquisition window for Target CTA mode scanning. Group CS image quality was significantly better than that for Group 75\% using both subjective and objective evaluations. The CS and CCTA diagnostic phases were both earlier than the empirically derived $75 \%$, with the diagnostic phases considered to be outside the $75 \%$-centered acquisition window in $19.5 \%$ of cases. The premise of this study of correlation between the CS and CTCAG diagnostic phases was also proven. The correlation efficiency between diagnostic phases of CS and CCTA was $0.351(p=0.02)$ indicating weak positive correlation [20].

The greatest advantage of adjusting the center of the acquisition window using CS instead of applying a fixed percentage as the center of acquisition window was an improvement in image quality due to individual adjustments made to the center of the acquisition window. The necessity of this adjustment is based on the wide individual variation in the diagnostic phase and the significant correlation between CS and CCTA scan diagnostic phases. The major disadvantage of this method is the increased workload during the scan as the method requires several additional steps, as compared with using a fixed percentage. CS images need to be reconstructed with a narrower field of view, the diagnostic phase must be identified on multiple planes using cardiacphase search software and must be reconstructed using the searched phase. This entire sequence of actions needs to be completed in timely fashion (i.e., before the CCTA scan) and 
TABle 1: Patient demographics.

\begin{tabular}{lccc}
\hline Parameter & Group 75\% & Group CS & $p$ value \\
\hline Number of patients & 40 & 41 & \\
Male/female & $28 / 12$ & $21 / 20$ & 0.14 \\
Age (years) & $66.8 \pm 11.8$ & $66.6 \pm 9.9$ & 0.94 \\
Body weight $(\mathrm{kg})$ & $64.1 \pm 14.8$ & $60.7 \pm 11.5$ & 0.26 \\
Body mass index $\left(\mathrm{kg} / \mathrm{m}^{2}\right)$ & $24.4 \pm 3.8$ & $24.2 \pm 3.9$ & 0.83 \\
Beta-blocker $^{+}$ & $15(38)$ & $19(46)$ & 0.28 \\
Heart rate $(\mathrm{bpm})$ & $58.9 \pm 6.5$ & $57.7 \pm 7.0$ & 0.44 \\
Coronary risk factor & & & \\
$\quad$ Hypertension & $21(53)$ & $26(63)$ & 0.18 \\
$\quad$ Diabetes mellitus & $10(25)$ & $12(29)$ & 0.43 \\
$\quad$ Dyslipidemia & $21(53)$ & $25(61)$ & 0.29 \\
$\quad$ Smoking & $17(43)$ & $17(41)$ & 0.55 \\
Family history & $3(8)$ & $5(12)$ & 0.37 \\
\hline
\end{tabular}

${ }^{+}$Data represent the number of patients (percentage).

TABLE 2: Scanning parameters.

\begin{tabular}{lccc}
\hline Parameter & Group 75\% & Group CS & $p$ value \\
\hline Contrast medium $(\mathrm{mL})$ & $45.5 \pm 9.4$ & $42.8 \pm 8.0$ & 0.17 \\
Injection rate $(\mathrm{mL} / \mathrm{sec})$ & $3.8 \pm 0.8$ & $3.6 \pm 0.6$ & 0.13 \\
Tube current $(\mathrm{mA})$ & $397 \pm 125$ & $354 \pm 92$ & 0.08 \\
Scan length $(\mathrm{cm})$ & $13.1 \pm 1.3$ & $12.9 \pm 1.2$ & 0.47 \\
Effective dose $(\mathrm{mSv})$ & $1.87 \pm 0.75$ & $1.70 \pm 0.66$ & 0.28 \\
\hline
\end{tabular}

TABLE 3: Comparison of reconstruction phases between both reconstruction methods.

\begin{tabular}{lccc}
\hline & Group 75\% & Group CS & $p$ value \\
\hline CS scan (\%) & & & \\
$\quad$ Average & 75 (unadjustable) & $73.9 \pm 3.0$ & N/A \\
Range & N/A & $67.0-85.3$ & \\
CCTA scan (\%) & & & \\
$\quad$ Average & $75.7 \pm 3.2$ & $73.6 \pm 4.5$ & $0.013^{*}$ \\
Range & $70.2-81.2$ & $60.8-82.0$ & \\
\hline
\end{tabular}

${ }^{*}$ Statistically significant.

TABLE 4: Subjective image quality.

\begin{tabular}{lccc}
\hline & Group 75\% & Group CS & $p$ value \\
\hline Overall & $3.20 \pm 0.66$ & $3.58 \pm 0.63$ & $<0.0001^{*}$ \\
RCA & $3.18 \pm 0.65$ & $3.63 \pm 0.60$ & $<0.0001^{*}$ \\
LMT + LAD + HL & $3.23 \pm 0.66$ & $3.58 \pm 0.62$ & $<0.0001^{*}$ \\
LCX & $3.17 \pm 0.67$ & $3.55 \pm 0.67$ & $<0.0001^{*}$ \\
\hline
\end{tabular}

${ }^{*}$ Statistically significant.

RCA: right coronary artery (\#1-4 and \#16).

LMT + LAD + HL: left main trunk, left anterior descending, and high lateral branch (\#5-10 and \#17).

LCX: left circumflex artery (\#11-15 and \#18).

must be repeated for certain "difficult" cases. Therefore, the phase search usually requires the input of a radiologist or a technologist in addition to the scanning technologist.

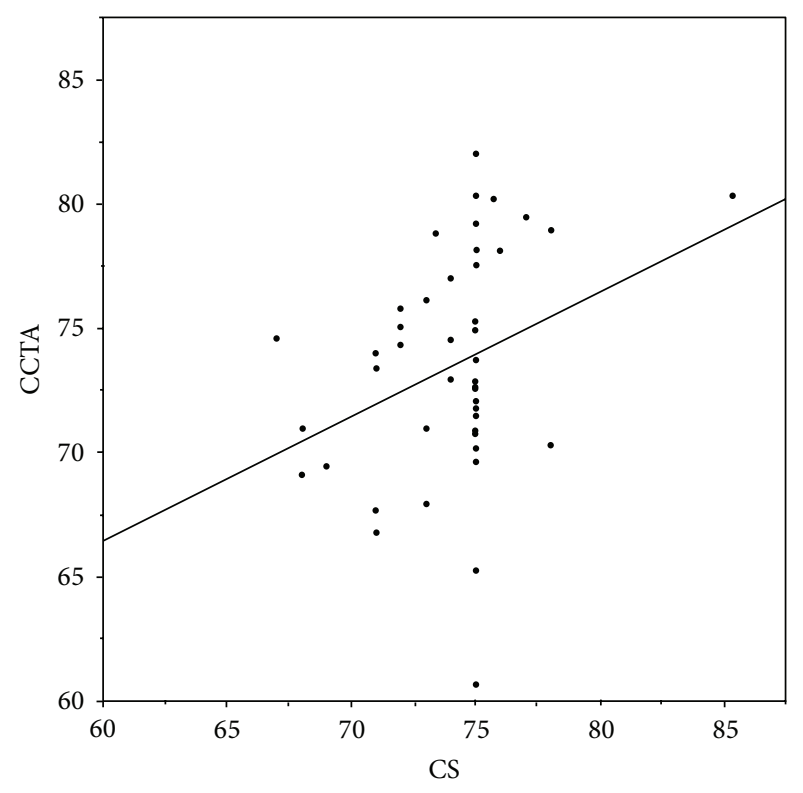

FIGURE 2: Scatter plot comparing the calcium scoring (CS) scan and coronary computed tomography angiography (CCTA) diagnostic phases for each patient. The line represents predictive formula for the CCTA diagnostic phase, which is CCTA diagnostic phase = $36.3+0.5 \times$ CS diagnostic phase.

TABLE 5: Objective image quality.

\begin{tabular}{lccc}
\hline & Group 75\% & Group CS & $p$ value \\
\hline Signal-to-noise ratio & & & \\
$\quad$ Overall & $21.5 \pm 2.0$ & $21.5 \pm 2.1$ & 0.98 \\
RCA & $20.6 \pm 4.6$ & $13.4 \pm 3.1$ & $<0.0001^{*}$ \\
LAD & $21.1 \pm 4.3$ & $14.8 \pm 2.8$ & $<0.0001^{*}$ \\
LCX & $19.7 \pm 3.3$ & $16.1 \pm 3.0$ & $0.0038^{*}$ \\
Contrast-to-noise ratio & & & \\
Overall & $25.2 \pm 5.7$ & $23.1 \pm 4.0$ & 0.24 \\
RCA & $27.5 \pm 5.4$ & $40.7 \pm 12.5$ & $0.0023^{*}$ \\
LAD & $26.9 \pm 7.0$ & $35.6 \pm 9.9$ & $0.015^{*}$ \\
LCX & $26.2 \pm 6.5$ & $31.4 \pm 9.4$ & $0.112^{*}$ \\
\hline${ }^{*}$ Statistically significant. & & &
\end{tabular}

There is a worldwide trend to reduce radiation exposure during cardiac CT. Indeed, radiologists should make their best effort to achieve "as low as reasonable achievable (ALARA)" radiation exposure during every examination. Target CTA scan is a product of the response to this mandate. However, the present study showed wide individual variation in cardiac CT diagnostic phase. Radiologists should tailor the center of the acquisition window during Target CTA scanning or set the acquisition window wider than the narrowest setting to help achieve this goal. For instance, Steigner et al. suggest $72-81 \%$ acquisition window has a good probability of including the diagnostic phase for $95 \%$ coronary arteries [21]. If Target CTA is to be used without tailoring the center of acquisition window using CS, the accompanying physician or technologist should at least look for motion artifacts on the CS scan before deciding to use Target CTA for CCTA 


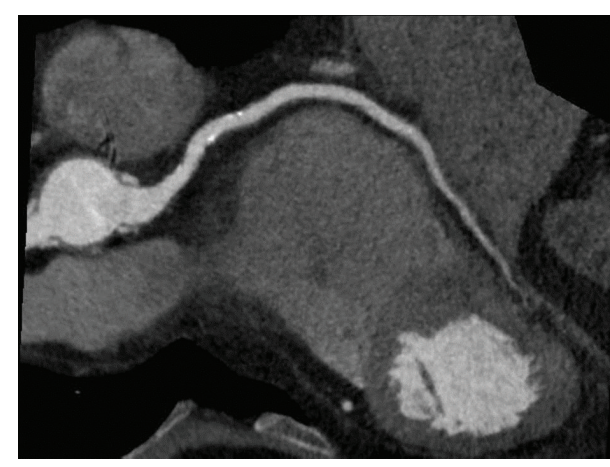

(a)

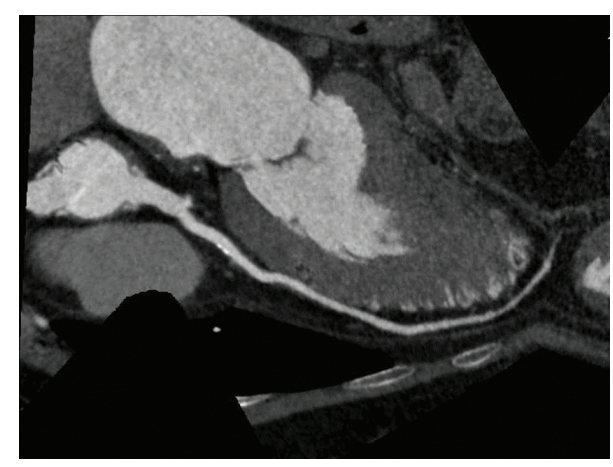

(b)

FIGURE 3: Presurgical screening coronary computed tomography angiography (RR interval = $1114 \mathrm{~ms})$ performed in Target CTA mode on a 61-year-old male with a history of aortic valve replacement. Curved multiplanar reconstruction images show the right coronary (a) and left anterior descending arteries (b) with minimal motion artifacts. The diagnostic phase was $68 \%$ for calcium scoring scan and $69.1 \%$ for coronary computed tomography angiography. The virtual window of $75 \%$ exposure was calculated as $70.0-80.4 \%$, which does not include the CCTA diagnostic phase.

scanning. If coronary arteries on the CS images contain motion artifacts, setting a wider acquisition window than the Target CTA (e.g., 70-80\%) raises the probability of obtaining better CCTA images without motion artifacts.

There are some limitations to our study. The heart usually straddles two volumes in CS, because CS needs to cover an area wider than the heart. First, because the RR intervals differ between those volumes, the reconstruction window becomes narrow when there is a large difference in the RR intervals. When this difference is too big, the reconstruction requires an artificial adjustment of the position of one of the $\mathrm{R}$ waves. In this study, cases that required such adjustment were considered to be arrhythmic and were excluded, because a longer CCTA acquisition window was applied to such cases. For the second limitation, the center of the CS acquisition window was fixed to $75 \%$. This means that if the diagnostic phase existed at an extreme such as $55 \%$ or $92 \%$, it would not be possible to include this phase, even when the center of the acquisition window was adjusted using CS. In fact, with some of our cases, the CS diagnostic phase was located at the earliest pole of the scan. Likewise, the best CCTA reconstruction phase was also found at the earliest pole. With these cases, there is likely to be and even better phase that occurred even earlier. The third study limitation was that many cases exhibited a gap between the CS and CCTA diagnostic phases, as was expected based on the correlation coefficient. In cases with large gaps, an even better phase may exist beyond the CCTA acquisition window. The fourth limitation pertains to the limited sample size because the average Group CS diagnostic phases and the percentage of patients that had values outside of the $75 \%$ fixed scan acquisition window were derived from a small number of patients. This point could be addressed by repeating the investigation in a larger population. In addition, further studies should be performed to determine whether the method described herein is also effective with other CT systems, such as 256-row CT or dualsource CT.

\section{Conclusions}

This study found that CS and CCTA diagnostic phases were significantly correlated, with average diagnostic phase of $73.9 \%$ and $73.6 \%$, respectively, although the phases showed substantial interindividual variation. The CCTA scan image quality using Target CTA mode was significantly better when the center of the acquisition window was adjusted using CS, compared with that using a fixed percentage.

\section{Conflict of Interests}

Rumiko Torigoe is a regular employee of Toshiba Medical Systems Corporation. The other authors have no conflict of interests regarding the publication of this paper.

\section{References}

[1] M. J. Budoff, S. Achenbach, R. S. Blumenthal et al., "Assessment of coronary artery disease by cardiac computed tomography: a scientific statement from the American Heart Association Committee on Cardiovascular Imaging and Intervention, Council on Cardiovascular Radiology and Intervention, and Committee on Cardiac Imaging, Council on Clinical Cardiology," Circulation, vol. 114, pp. 1761-1791, 2006.

[2] D. B. Mark, D. S. Berman, M. J. Budoff et al., "ACCF/ ACR/AHA/NASCI/SAIP/SCAI/SCCT 2010 expert consensus document on coronary computed tomographic angiography: a report of the American College of Cardiology Foundation Task Force on Expert Consensus Documents," Circulation, vol. 121, pp. 2509-2543, 2010.

[3] A. J. Taylor, M. Cerqueira, J. M. Hodgson et al., "ACCF/SCCT/ ACR/AHA/ASE/ASNC/NASCI/SCAI/SCMR 2010 appropriate use criteria for cardiac computed tomography: a report of the American College of Cardiology Foundation Appropriate Use Criteria Task Force, the Society of Cardiovascular Computed Tomography, the American College of Radiology, the American 
Heart Association, the American Society of Echocardiography, the American Society of Nuclear Cardiology, the North American Society for Cardiovascular Imaging, the Society for Cardiovascular Angiography and Interventions, and the Society for Cardiovascular Magnetic Resonance," Journal of the American College of Cardiology, vol. 56, no. 22, pp. 1864-1894, 2010.

[4] M. J. Budoff, D. Dowe, J. G. Jollis et al., "Diagnostic performance of 64-multidetector row coronary computed tomographic angiography for evaluation of coronary artery stenosis in individuals without known coronary artery disease: results from the prospective multicenter ACCURACY (Assessment by Coronary Computed Tomographic Angiography of Individuals Undergoing Invasive Coronary Angiography) trial," Journal of the American College of Cardiology, vol. 52, no. 21, pp. 1724-1732, 2008.

[5] J. M. Miller, C. E. Rochitte, M. Dewey et al., "Diagnostic performance of coronary angiography by 64-row CT," The New England Journal of Medicine, vol. 359, no. 22, pp. 2324-2336, 2008.

[6] A. Arbab-Zadeh, J. M. Miller, C. E. Rochitte et al., "Diagnostic accuracy of computed tomography coronary angiography according to pre-test probability of coronary artery disease and severity of coronary arterial calcification: the CORE-64 (Coronary Artery Evaluation Using 64-Row Multidetector Computed Tomography Angiography) international multicenter study," Journal of the American College of Cardiology, vol. 59, no. 4, pp. 379-387, 2012.

[7] N. Tomizawa, S. Kanno, E. Maeda, M. Akahane, R. Torigoe, and K. Ohtomo, "Minimizing the acquisition phase in coronary CT angiography using the second generation 320-row CT," Japanese Journal of Radiology, vol. 32, no. 7, pp. 391-396, 2014.

[8] M. Y. Chen, S. M. Shanbhag, and A. E. Arai, "Submillisievert median radiation dose for coronary angiography with a secondgeneration 320-detector row CT scanner in 107 consecutive patients," Radiology, vol. 267, no. 1, pp. 76-85, 2013.

[9] F. Khosa, A. Khan, K. Nasir et al., "Influence of image acquisition on radiation dose and image quality: full versus narrow phase window acquisition using 320 MDCT,' The Scientific World Journal, vol. 2013, Article ID 731590, 5 pages, 2013.

[10] S. Fujimoto, H. Matsutani, T. Kondo et al., "Image quality and radiation dose stratified by patient heart rate for coronary 64 and 320-MDCT angiography," American Journal of Roentgenology, vol. 200, no. 4, pp. 765-770, 2013.

[11] D. Andreini, G. Pontone, S. Mushtaq et al., "Coronary stent evaluation with coronary computed tomographic angiography: comparison between low-osmolar, high-iodine concentration iomeprol-400 and iso-osmolar, lower-iodine concentration iodixanol-320," Journal of Cardiovascular Computed Tomography, vol. 8, no. 1, pp. 44-51, 2014.

[12] Y. Funama, D. Utsunomiya, K. Taguchi, S. Oda, T. Shimonobo, and Y. Yamashita, "Automatic exposure control at single- and dual-heartbeat CTCA on a 320-MDCT volume scanner: effect of heart rate, exposure phase window setting, and reconstruction algorithm," Physica Medica, vol. 30, no. 3, pp. 385-390, 2014.

[13] F. Tatsugami, T. Higaki, W. Fukumoto et al., "Radiation dose reduction for coronary artery calcium scoring at 320-detector CT with adaptive iterative dose reduction 3D," The International Journal of Cardiovascular Imaging, vol. 31, no. 5, pp. 1045-1052, 2015.

[14] J. Hausleiter, T. Meyer, F. Hermann et al., "Estimated radiation dose associated with cardiac CT angiography," The Journal of the American Medical Association, vol. 301, no. 5, pp. 500-507, 2009.
[15] M. Y. Chen, M. L. Steigner, S. W. Leung et al., "Simulated $50 \%$ radiation dose reduction in coronary CT angiography using adaptive iterative dose reduction in three-dimensions (AIDR3D)," International Journal of Cardiovascular Imaging, vol. 29, no. 5, pp. 1167-1175, 2013.

[16] A. Bedayat, F. J. Rybicki, K. Kumamaru et al., "Reduced exposure using asymmetric cone beam processing for wide area detector cardiac CT,' International Journal of Cardiovascular Imaging, vol. 28, no. 2, pp. 381-388, 2012.

[17] G. L. Raff, A. A. Chair, S. Achenbach et al., "SCCT guidelines for the interpretation and reporting of coronary computed tomographic angiography," Journal of Cardiovascular Computed Tomography, vol. 3, no. 2, pp. 122-136, 2009.

[18] N. Tomizawa, S. Komatsu, M. Akahane, R. Torigoe, S. Kiryu, and K. Ohtomo, "Relationship between beat to beat coronary artery motion and image quality in prospectively ECG-gated two heart beat 320-detector row coronary CT angiography," International Journal of Cardiovascular Imaging, vol. 28, no. 1, pp. 139-146, 2012.

[19] N. Tomizawa, T. Nojo, M. Akahane, R. Torigoe, S. Kiryu, and K. Ohtomo, "Adaptive Iterative Dose Reduction in coronary CT angiography using 320-row CT: assessment of radiation dose reduction and image quality," Journal of Cardiovascular Computed Tomography, vol. 6, no. 5, pp. 318-324, 2012.

[20] M. M. Mutaka, "A guide to appropriate use of correlation coefficient in medical research," Malawi Medical Journal, vol. 24, no. 3, pp. 69-71, 2012.

[21] M. L. Steigner, H. J. Otero, T. Cai et al., "Narrowing the phase window width in prospectively ECG-gated single heart beat 320-detector row coronary CT angiography," International Journal of Cardiovascular Imaging, vol. 25, no. 1, pp. 85-90, 2009. 


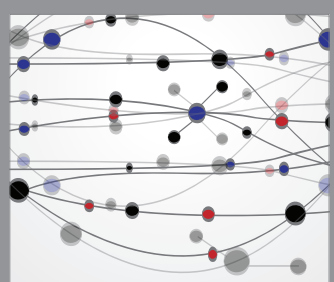

The Scientific World Journal
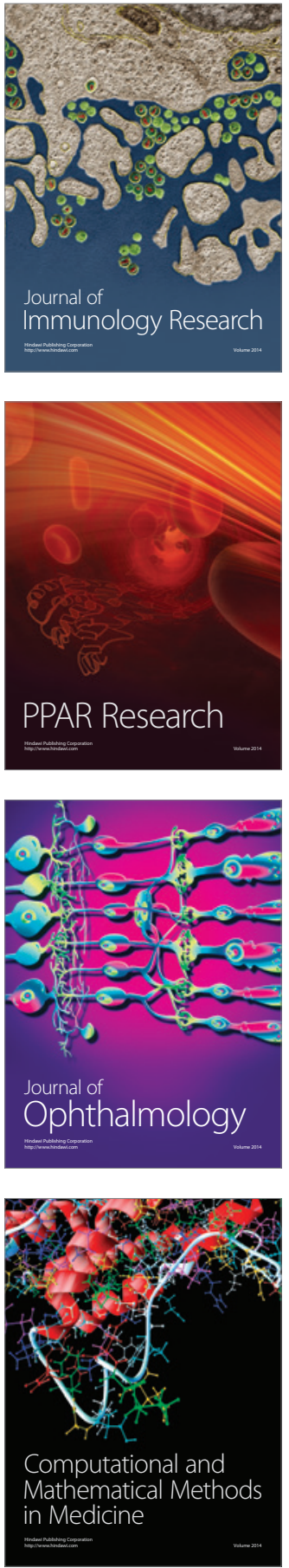

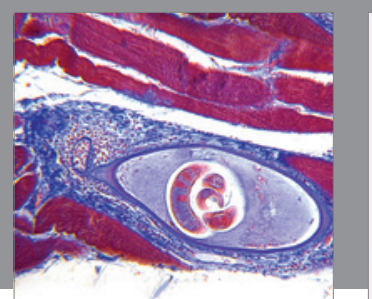

Gastroenterology Research and Practice

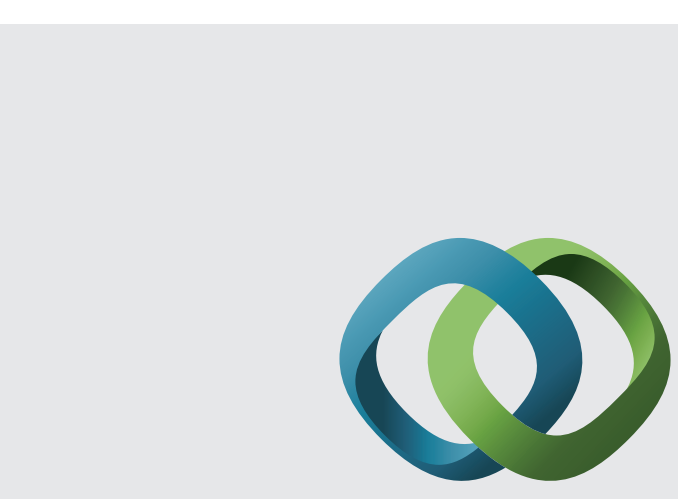

\section{Hindawi}

Submit your manuscripts at

http://www.hindawi.com
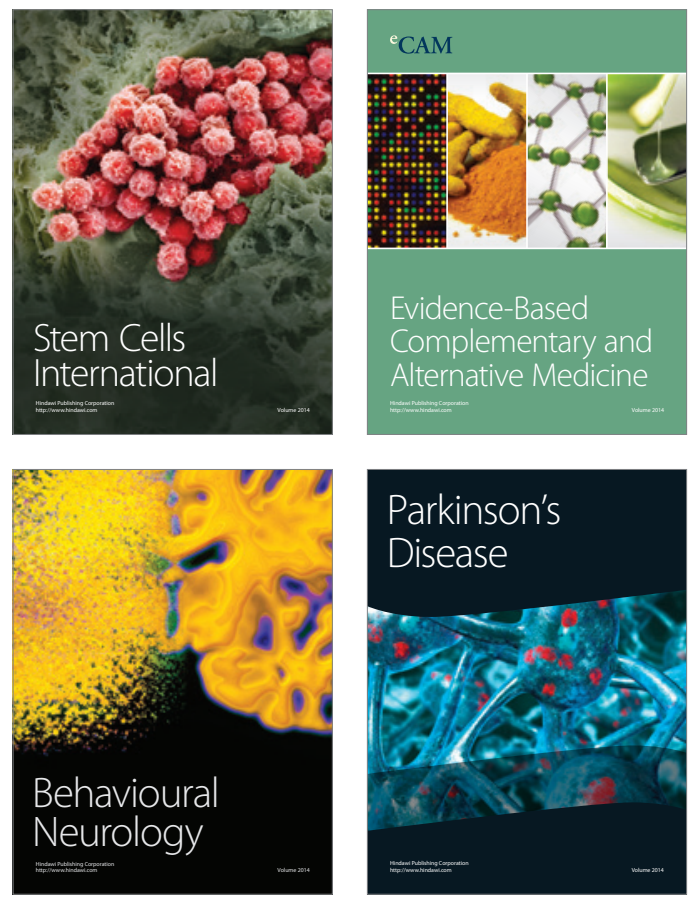
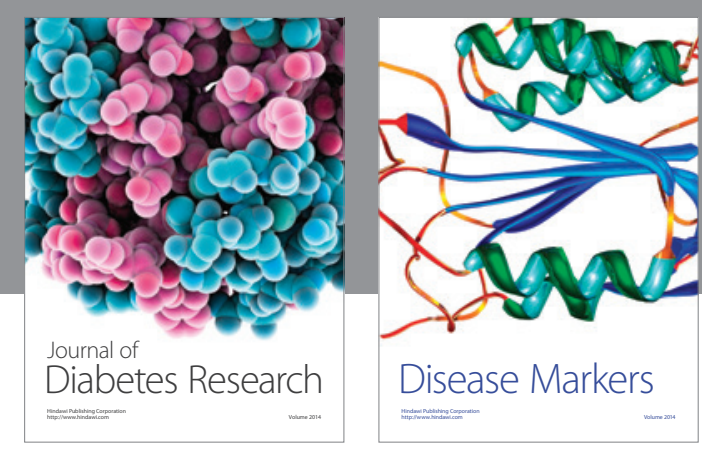

Disease Markers
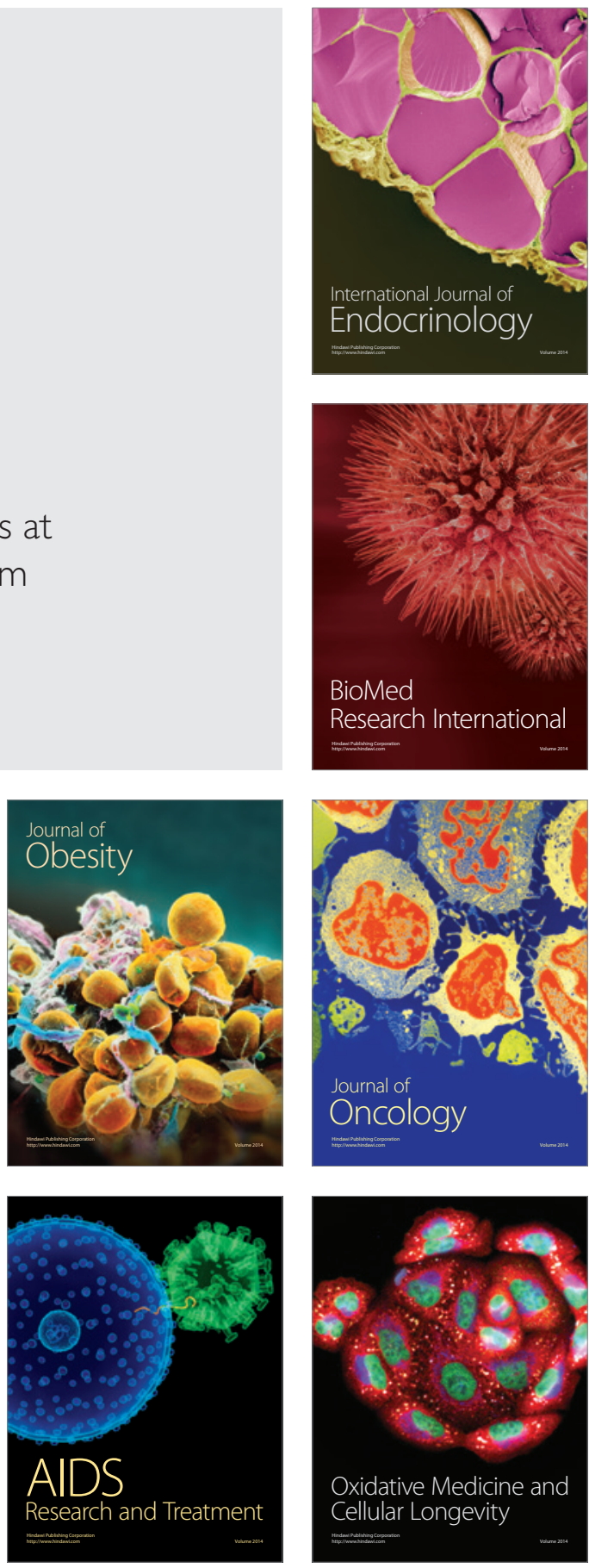